\title{
On the nature of interlayer interactions in a system of two graphene fragments
}

\author{
Julia Berashevich and Tapash Chakraborty \\ Department of Physics and Astronomy, University of Manitoba, Winnipeg, Canada, R3T 2N2
}

\begin{abstract}
With the help of the quantum chemistry methods we have investigated the nature of interlayer interactions between graphene fragments in different stacking arrangements (AA and $\mathrm{AB})$. We found that the $\mathrm{AB}$ stacking pattern as the ground state of the system, is characterized by the effective inter-band orbital interactions which are barely present in the AA. Their vanishing induces electronic decoupling between the graphene layers, so that the bonding interaction $\Delta E_{o i}$ between the flakes is drastically reduced from $-0.482 \mathrm{eV}$ to $-0.087 \mathrm{eV}$ as the stacking pattern is changed from $\mathrm{AB}$ to AA. The effective way to improve the bonding interaction between layers preserving the same AA lattice order is to induce rotation of the layer. As the flake is rotated, the bonding interactions are improved mostly due to suppression of the Pauli repulsion which in turn increases the interlayer orbital interactions, while the inter-band part of those remain negligible on the whole range of the rotation angle. The Pauli repulsion is also found to be the main force moving apart two fragments as the stacking pattern is changed from AA to AB. This enhances the equilibrium interlayer distance, which for the AA staking is larger than the established value for the AB stacking $(3.4 \AA)$.
\end{abstract}

Bilayer graphene [1 7], being regarded as an important system for applications in semiconductor electronics has intrigued the scientific community to look deeper into the nature of interlayer interactions in this system. Graphene layers stacked together is uniquely different from other solid state materials because of the interlayer weak van der Waals and steric interactions, instead of the occurrence of $\mathrm{C}-\mathrm{C}$ bonding between the layers. The half-filled $p$ orbital left on each carbon atom after bonding with its neighbors within the honeycomb lattice is responsible for formation of the $\pi$ bonds between two neighboring atoms within the same layer. This creates a closed shell electron system carrying the weakly bound $\pi$ electrons that are distinct due to their high mobility within the graphene layer [8-10]. Stacking two systems of closed electron shells would cause the interlayer interactions to be mostly repulsive which results in the expulsion of valence electrons from the overlap region such that only a weak electronic coupling between the layers can occur. If under certain conditions the electronic coupling becomes negligible then each layer would display its own electronic behavior in the band diagram as was found recently in twisted bilayer graphene [11 13$]$.

Even before the discovery of graphene the interlayer interactions in natural graphite, which is basically a system of stacked graphene layers, received intense attention [14-17] where the effect of interlayer decoupling was not encountered. Three types of layer arrangements are known to exist in graphite [14], but the most common one is the Bernal stacking in which the carbon atoms belonging to different sublattices $\mathrm{A}$ and $\mathrm{B}$ form the $\mathrm{AB}$ stacking pattern between the layers. Contrary to the conventional wisdom that only the long-range van der Waals interaction is important in the case of stacking of closed shell systems, for the $\mathrm{AB}$ stacking in graphite it was shown that the orbital overlap between the $\pi$ orbitals belonging to different layers [15, 18] is as essential as the all important van der Waals forces. Therefore, it was predicted that despite the well known interlayer distance of $3.4 \AA$ in natural graphite, for a system containing only two or three layers, the interlayer spacing depends on the number of layers [15] due to different nodal interactions of the overlapping $\pi$ orbitals. For an odd number of adjacent layers the equilibrium spacing between the layers was predicted to be $3.30 \AA$, while for the even number it is 3.58 $\AA$ [15]. It then clearly follows that in graphite the electronic coupling between the layers can not be neglected. Rather, it provides a substantial influence on the interlayer interactions in addition to the van der Walls and steric type of interactions.

In this context, it is worth pondering what is acually happening with the interlayer interactions in twisted bilayer graphene where interestingly, one observes electronic decoupling between the layers. The decoupling was first observed experimentally [1-3] and was later investigated theoretically $11-13$. Theoretical interpretations [11 13] relate the decoupling to the occurrence of a misorientation of $2^{\circ}-5^{\circ}$ between the layers. According to a proposed model, the layer rotation in the real space induces a displacement of the Dirac cones generated in each layer in the reciprocal space [11-13] thereby causing the interlayer decoupling. Experimentally, the misorientation of $2^{\circ}-5^{\circ}$ in AA-stacked bilayer graphene has been detected in systems that were created using various fabrication techniques such as the epitaxial growth [1-3], chemical vapor deposition [4] and ultrasonication 5]. Appearance of the rotational misorientation that is independent of the fabrication techniques suggests the presence of some forces between the layers strong enough to cause the layer rotation. However, the available theoretical models [11 13] on the electronic properties of the AA stacked graphene deal only with the band properties but do not shed any light on the underlying physical reasons involved in decoupling, such as the interlayer forces.

In a recent work [18] we related the origin of the decoupling phenomenon and rotational misorientation with layer stacking pattern which is AA in fabricated multilayer graphene [1 [5] against the $\mathrm{AB}$ stacking in natural graphite. For the AA staking, the interlayer electronic coupling is suppressed by a significant repulsion arising 
between the graphene layers [18]. This repulsion is also expected to be responsible for the occurrence of lattice misorientation between the layers. It was suggested that rotational misorientation, which creates the Moiré pattern, appears as a way to suppress the repulsion, thereby lowering the total energy of the system. Even a slight layer rotation of $\sim 2^{\circ}-5^{\circ}$ substantially shrinks the areas characterized by the AA lattice superposition in which repulsion dominates over other forces (the areas with AA and $\mathrm{AB}$ stacking coexist in the Moiré pattern). The other important result was a prediction [18] that the strong repulsion may induce bumps on the graphene surface in the areas where AA stacking is preserved. All these effects are important and require careful studies because the phenomenon of layer rotation through electronic coupling between layers can offer ways to manipulate the electronic properties of twisted graphene (Moiré pattern of different rotation angle is characterized by different percentage of AA-spotted areas). Therefore, in this work we present a detailed quantitative analysis of the repulsive forces and the orbital overlap in stacked graphene layers and their alteration with the appearance of rotation. Our studies are based on the density functional methods including a recently proposed empirical correction (Grimme correction [19]) which was developed for a proper consideration of the dispersive interactions between the closed shell electron systems.

\section{COMPUTATIONAL METHODS}

The computations were performed with the ADF quantum chemistry code [20] which uses the Kohn-Sham approach to density functional theory (DFT). The KohnSham approach replaces the many-body system within the Hamiltonian equation by a system of the noninteracting particles while all the many body terms are incorporated into the so-called Kohn-Sham potential. This concept is quite useful in the investigation of interacting closed shell systems because it allows us to present each graphene flake as an isolated fragment and two fragments interacts as the flakes are stacked. In this way, a proper investigation of the forces and the orbital overlap can be performed directly in terms of the fragment presentation.

Within the ADF code the forces between fragments are included in the bonding energy $\Delta E^{0}$ which comprises of several majors components [21] $\left(\Delta E^{0}=\right.$ $\left.\Delta V_{e l}+\Delta E_{p}+\Delta E_{\text {prep }}+\Delta E_{o i}+E_{\text {dis }}\right)$. The first component $\left(\Delta V_{e l}\right)$ takes care of the interactions of electrostatic nature related to the modification of the charge distribution (originated from the charge transfer between occupied and unoccupied orbitals), when two systems are allowed to interact. The second one is the energy change induced by the Pauli repulsion $\left(\Delta E_{p}\right)$, which include several components; exchange repulsion, kinetic repulsion, overlap repulsion, all results from obeying the Pauli antisymmetry principle. The next term $\Delta E_{\text {prep }}$ describes the energy required to change the conformation of the fragments (structural modification) from the initial geometry containing separate fragments to the final geometry where the fragments are allowed to interact. The bonding interactions between two fragments are included in the $\Delta E_{o i}$ term which originates from the overlap of the fragment's orbitals. The last term $E_{d i s}$ is the empirical dispersion correction introduced by Grimme [19] and its magnitude is defined by the long-range van der Waals interactions, whose contribution in the short range is reduced by the damping function.

Even though the $\Delta E_{o i}$ term is a measure of the orbital overlap, the interlayer forces such as the Pauli repulsion and orbital polarization contribute to the $\Delta E_{o i}$ as well. The effect of interlayer forces can not be discarded from $\Delta E_{o i}$ and so the overlap of the selected orbitals can not be separated from the others. This makes it hard to get a proper understanding of the intricacies of interlayer interactions between two fragments. The most effective way to proceed is to follow the established method of linear combinations of the orbitals. This method can be applied for the results obtained with the ADF program. With the ADF, each fragment is described by its own set of orbitals and the program facilitates their mixing upon the inclusion of the interaction between the fragments. Therefore, the fragment approach allows us to evaluate the overlap matrix $S_{i, j}$ between the fragments $i$ and $j$ of the Kohn-Sham Hamiltonian $\left(\left\langle\varphi_{i}\left|h_{\mathrm{KS}}\right| \varphi_{j}\right\rangle\right)$ directly in terms of the linear combinations of the fragment orbitals via the relation $h_{\mathrm{KS}}=S C E C^{-1}$, where $C$ is the eigenvector defined in terms of the fragment orbitals and $E$ is the eigenvalue matrix [22]. The overlap matrix $S$ purely depends on the form of the interacting orbitals and on the distance that keeps the two fragments apart neglecting the contribution from the attractive and repulsive forces arising between the fragments. We used the overlap matrix $S_{i, j}$ to define the spatial overlap integral between the fragments $i$ and $j$, which is $J_{i, j}=\left\langle\varphi_{i}|H| \varphi_{j}\right\rangle$.

In this work we consider the spatial overlap integral $J_{i, j}^{H-H}$ between the highest occupied fragment orbitals (HOFO), i.e., between two $\pi$ orbitals, each located on different fragments while their overlap defines the HOMO of the joint system. The overlap integral was also calculated between the $\pi$ and $\pi^{*}$ orbitals, i.e., between the highest occupied orbital of one fragment $\left(\mathrm{HOFO}_{1}\right)$ with the lowest unoccupied orbital of another fragment $\left(\mathrm{LUFO}_{2}\right)$ and because there are two parts of such interactions, $\mathrm{HOFO}_{1^{-}}$ $\mathrm{LUFO}_{2}$ and $\mathrm{HOFO}_{2}-\mathrm{LUFO}_{1}$, the average value of overlap integral was considered and combined into the $J_{i, j}^{H-L}$.

We used the hybrid BLYP exchange-correlation functional, applying the empirical dispersion correction 1.05 recommended by Grimme [19, 23]. For the interacting molecules of closed electron shells it was found that the proposed correction is enough to reproduce the intermolecular distance to what is observed in the experiments or achieved with a more accurate level such as the $a b i n i-$ tio Møller-Plesset second-order (MP2) method [19]. For a proper description of the tails of the electron wave- 
functions that is important for long-range interactions, we used the Slater-type orbitals. The quite extended TZP basis set (triple- $\zeta$ polarized basis set) was applied in all the calculations which improves the precision of our results while suppressing the basis set superposition error [24]. We tested the chosen method to reproduce the interlayer distance between the graphene flakes stacked in the $\mathrm{AB}$ pattern (which is well known to be $3.4 \AA$ in natural graphite) and indeed the correct interlayer distance was obtained (the so-called equilibrium distance of the $\mathrm{AB}$ pattern $\left.d_{e q(A B)}\right)$. For this calculation the atomic coordinates within the graphene plane (along the $x, y$ directions) were confined and only the coordinates in the $z$ direction, i.e., perpendicular to the graphene plane, were used for relaxation. In fact, the full relaxation of the system of two stacked fragments is problematic as the repulsion between layers leads to sliding of the fragments away from each other.

Our main emphases in this work are the energy decomposition analysis of the bonding energy $\Delta E^{0}\left(\Delta V_{e l}\right.$, $\Delta E_{p}, \Delta E_{\text {prep }}$ and $\left.\Delta E_{o i}\right)$ and the spatial overlap integrals $\left(J_{i, j}^{H-H}, J_{i, j}^{H-L}\right)$ which are determined by the stacking pattern between two graphene flakes (flake rotation) and the interlayer distance. In most cases the single point calculations have been used for which the contribution of $\Delta E_{\text {prep }}$ becomes zero.

\section{AA AND AB STACKING}

For our investigations we used two graphene flakes with the carbon atoms at the edges terminated by the hydrogen atoms, as shown in Fig. 1 (a) with our goal to minimize the contribution of the localized states into the simulation results. Because bilayer graphene obtained in the experiments [1 3] has shown the AA stacking pattern instead of the $\mathrm{AB}$, common in natural graphite, we probe the AA stacking for the equilibrium distance between the flakes $\left(d_{e q(A A)}\right)$. It was found that this distance is indeed enhanced in the AA stacking up to $d_{e q(A A)}=3.67$ $\AA$ against the $d_{e q(A B)}=3.4 \AA$ for the $\mathrm{AB}$ stacking. To explain an increase in the interlayer distance we applied the decomposition analysis of the bonding energy.

We performed the single point calculations in which the layers were separated by the established equilibrium distances $\left(d_{e q}\right)$ found to be different for $\mathrm{AA}$ and $\mathrm{AB}$ stacking. The obtained interlayer forces and spatial overlap integrals are collected in Table [. For the purpose of comparison, in addition to the lattice arrangements AA and $\mathrm{AB}$, we also carried out the bonding analysis for the $\mathrm{AA}^{\prime}$ stacking pattern for which the interlayer equilibrium distance was found to be $3.41 \AA$ and those results are also enclosed in Table II The AB-conformation is characterized by a much stronger bonding interaction than that of $\mathrm{AA}$, as defined by a more negative bonding energy $\Delta E^{0}$, thereby making the AB-stacking the ground state of the system. The structural distinction of the $\mathrm{AB}$ configuration from AA consists in sliding of one graphene flake
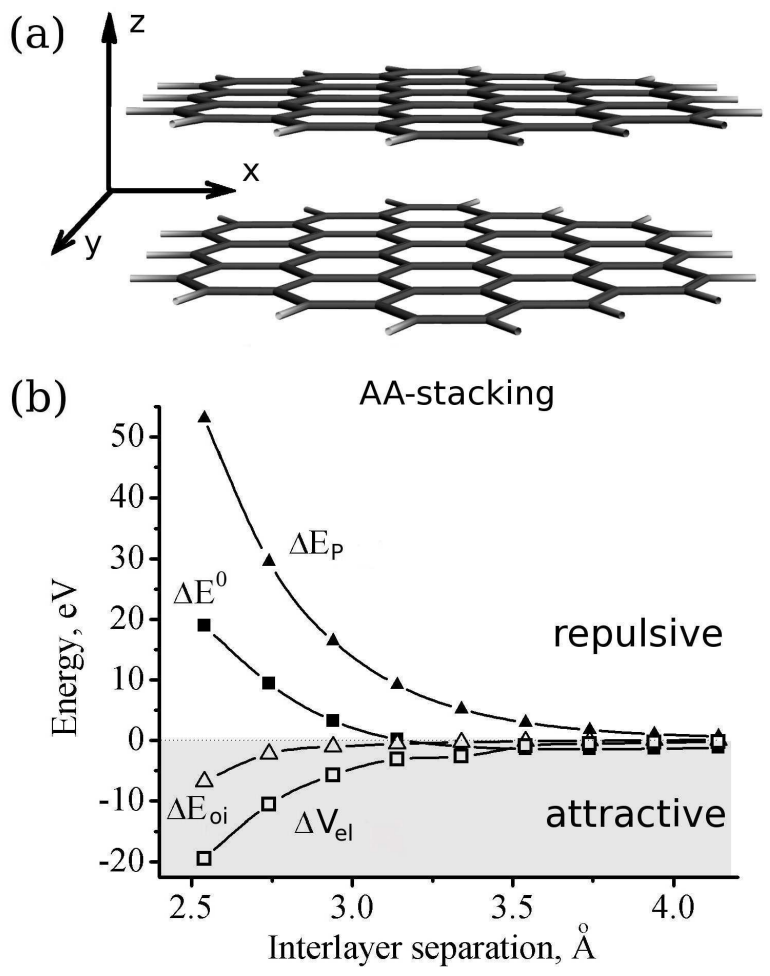

FIG. 1: (a) Stacked graphene flakes in the 3D space coordinate system. (b) The energy components $\left(\Delta V_{e l}, \Delta E_{p}, \Delta E_{o i}\right)$ of the bonding energy $\left(\Delta E^{0}\right)$ as a function of the interlayer distance between two graphene fragments. $\Delta V_{e l}$ is the electrostatic interactions, $\Delta E_{p}$ is Pauli repulsion, $\Delta E_{o i}$ is the orbital interactions energy.

relative to the other by $1.42 \AA$ along the $x$ axis that induces the shift of the bond positions between the layers against their superposition for the AA stacking (for the AA case the atomic coordinates are matched in the $x$ and $y$ directions for both layers). To obtain the $\mathrm{AA}^{\prime}$ stacking a sliding of $1.23 \AA$ is applied along the $y$ axis.

For the AA stacking, due to the lattice superposition the $\pi$ clouds between the layers are also superposed that leads to their effective overlap defined by the spatial overlap integral $J_{i, j}^{H-H}=0.443 \mathrm{eV}$. The flake sliding induced for the $\mathrm{AB}$ and $\mathrm{AA}^{\prime}$ stacking breaks the bond superposition condition recognized for the AA stacking thereby inducing the disarrangement of the $\pi$ clouds within the overlapping region. As a result, the $\pi-\pi$ interlayer interactions, which is of particular interest since it is supposedly responsible for the occurrence of electronic coupling between the graphene layers [7, 25], is significantly reduced for the $\mathrm{AB}$ stacking pattern. This is reflected by a suppression of the spatial overlap integral to $J_{i, j}^{H-H}=-0.229 \mathrm{eV}$ (even more drastic reduction is observed for the $\mathrm{AA}^{\prime}$ case where $J_{i, j}^{H-H}=0.023 \mathrm{eV}$ ).

When two fragments are stacked, the majority of the orbital interactions described by $\Delta E_{o i}$ arise from the overlap of the $\pi$ orbitals $\left(\pi-\pi\right.$ or $\left.\pi-\pi^{*}\right)$. For interaction of two closed shell systems, the $\pi-\pi$ overlap is not the 
TABLE I: The electronic properties and the interlayer forces between two graphene fragments stacked in AA $\left(d_{e q(A A)}=3.67\right.$ $\AA)$, in $\mathrm{AB}\left(d_{e q(A B)}=3.4 \AA\right)$ and in $\mathrm{AA}^{\prime}\left(d_{e q(A A)}=3.41 \AA\right)$ configurations. All values are in $\mathrm{eV}$.

\begin{tabular}{l|c|c|c|c|c|c|c|c}
\hline & HOMO & LUMO & $J_{i, j}^{H-H}$ & $J_{i, j}^{H-L}$ & $\Delta E_{o i}$ & $\Delta V_{e l}$ & $\Delta E_{p}$ & $\Delta E^{0}$ \\
\hline AA & -4.024 & -2.596 & 0.443 & $10^{-5}$ & -0.087 & -0.691 & 2.136 & -1.483 \\
AB & -4.164 & -2.506 & -0.229 & -0.169 & -0.482 & -1.184 & 3.388 & -1.931 \\
AA $^{\prime}$ & -4.070 & -2.565 & 0.023 & -0.191 & -0.491 & -1.263 & 3.644 & -1.863 \\
\hline
\end{tabular}

one that leads to the bonding interactions, and therefore, might be ignored within the orbital interaction term $\Delta E_{o i}$. That explains the contradictory behavior of the overlap integral $J_{i, j}^{H-H}$ and the $\Delta E_{o i}$ term, such that when a reduction of the overlap integral $J_{i, j}^{H-H}$ occurs for the $\mathrm{AB}$ stacking, the orbital interactions $\Delta E_{o i}$ between the fragments is improved. However, the improvement of $\Delta E_{o i}$ with modification of the lattice arrangement from $\mathrm{AA}$ to $\mathrm{AB}$ is also not consistent with behavior of the Pauli repulsion $\Delta E_{p}$ whose increase supposedly suppresses the interlayer orbital interaction $\Delta E_{o i}$. Therefore, to understand the alteration of the $\Delta E_{o i}$ term we should take into consideration other components of the orbital interactions, such as the orbital polarization and the interlayer interaction of the $\pi-\pi^{*}$ orbitals.

Orbital polarization reflects a mixing of the occupied/virtual orbitals in one fragment due to the presence of another fragment, i.e., each valence electron of one fragment entering the electron space of electrons of other fragment polarizes its orbitals. The polarization effect is caused by the repulsion arising between interacting electrons [26]. Analyzing the orbital formation after perturbation of the fragment's orbitals, a discrepancy in the product orbitals for the $\mathrm{AB}, \mathrm{AA}$ and $\mathrm{AA}^{\prime}$ stacking patterns was detected as demonstrated by the scheme in Fig. 2, Let us consider the formation of the orbitals generating the HOMO-LUMO gap in the joint system which would give the most contribution into the interlayer interaction of the $\pi-\pi^{*}$ orbitals. In formation of HOMO orbital (LUMO) of the joint system two degenerate fragment's orbitals HOFO participates (two LUFO orbitals for the LUMO formation). Perturbation of those degenerate HOFO orbitals (overall four in two fragments) creates the four molecular orbitals in the bilayer system (HOMO, HOMO-1, HOMO-2 and HOMO-3). Similarly, the LUFO orbitals perturb in the valence band so that two LUFOs are taken from each fragment and their perturbation leads to formation of four LUMO orbitals in the stacked system (LUMO, LUMO+1, LUMO+2, LUMO+3). For the AA stacking, two pairs of product orbitals possess an identical orbital energy, i.e., HOMO and $\mathrm{HOMO}-1$ (LUMO and LUMO+1) are degenerate, the same for $\mathrm{HOMO}-2$ and $\mathrm{HOMO}-3$ (LUMO+2 and $\mathrm{LUMO}+3)$. However, already for the $\mathrm{AB}$ case the conduction band is limited by a single HOMO orbital being a product of the perturbation of all four fragment's orbitals while the rest of the generated orbitals are shifted deeper into the conduction band where two of them still would remain degenerate. Mixing of the LUFOs for the $\mathrm{AB}$ stacking stays similar to those for the AA case, i.e., two pairs of the degenerate orbitals are formed. For the $\mathrm{AA}^{\prime}$ stacking all four product orbitals HOMOs (LUMOs) are separated by the energy gap.

The spatial orbital overlap $J_{i, j}^{H-H}$, regardless of the observed peculiarities of orbital mixing as lattice arrangement is changed, is being affected mostly by rearrangements of the $\pi$ clouds from their superposition in AA stacking. However, an analysis of interaction of the occupied/unoccupied orbitals between fragments $J_{i, j}^{H-L}$ has shown a distinct behavior. We observed barely present overlap between those orbitals in the AA pattern $\left(J_{i, j}^{H-L}=10^{-5} \mathrm{eV}\right)$, while it appears for the AB stacking to be $0.169 \mathrm{eV}$ and increases even further up to $0.191 \mathrm{eV}$ for the $\mathrm{AA}^{\prime}$ stacking. Such progress is consistent with improvement of the orbital interactions $\Delta E_{o i}$ and with enhancement of the attractive interactions of the electrostatic nature (see $\Delta V_{e l}$ in Table 【) as stacking pattern is changed from $\mathrm{AA}$ to $\mathrm{AB}$. Both these terms, $\Delta E_{o i}$ and $\Delta V_{e l}$, lowers the bonding energy $\Delta E^{0}$ and their contribution compensate the growing Pauli repulsion between fragments.

Therefore, the efficiency of the acceptor-donor interactions, defined by $\Delta E_{o i}$, is found to be several times (at least five) weaker for the AA stacking in comparison to that for the AB. This behavior offers an interpretation of the interlayer decoupling observed in the experiments for the AA stacking in twisted bilayer graphene [1 5], against the efficient coupling known for the AB stacking. According to our findings the interlayer decoupling in AA staking must be caused by suppression of the orbital overlap of the occupied, $\pi$, and unoccupied, $\pi^{*}$, orbitals between the fragments, i.e. inter-band (HOMO-LUMO) interaction, while variation of the $\pi-\pi$ overlap is found to have a insignificant affect on the interaction between fragments. This conclusion is, in fact, in contrast to the commonly accepted opinion that the electronic coupling between the stacked graphene layers, which is capable to change linear Dirac cones dispersion to parabolic one, originates from intra-band interlayer interactions [7, 25]. We should also note that along with the intra- and interband interactions the effect of orbital polarization is one of the significant contribution into the bonding interactions.

\section{INTERLAYER DISTANCE}

The observation that the interlayer repulsion characterized by $\Delta E_{p}$ is being stronger for the $\mathrm{AB}$ stacking in comparison to that for AA was beyond our expectations because the Pauli repulsion is presumed to dominate when one structure of closed electron shell is placed exactly on top of the other (such as the coordinates in $x-y$ directions coincide as presented in Fig. 11(a)). The Pauli repulsion has the exponential dependence on the separation distance and an increase in $d_{e q}$ for the $\mathrm{AA}$ 

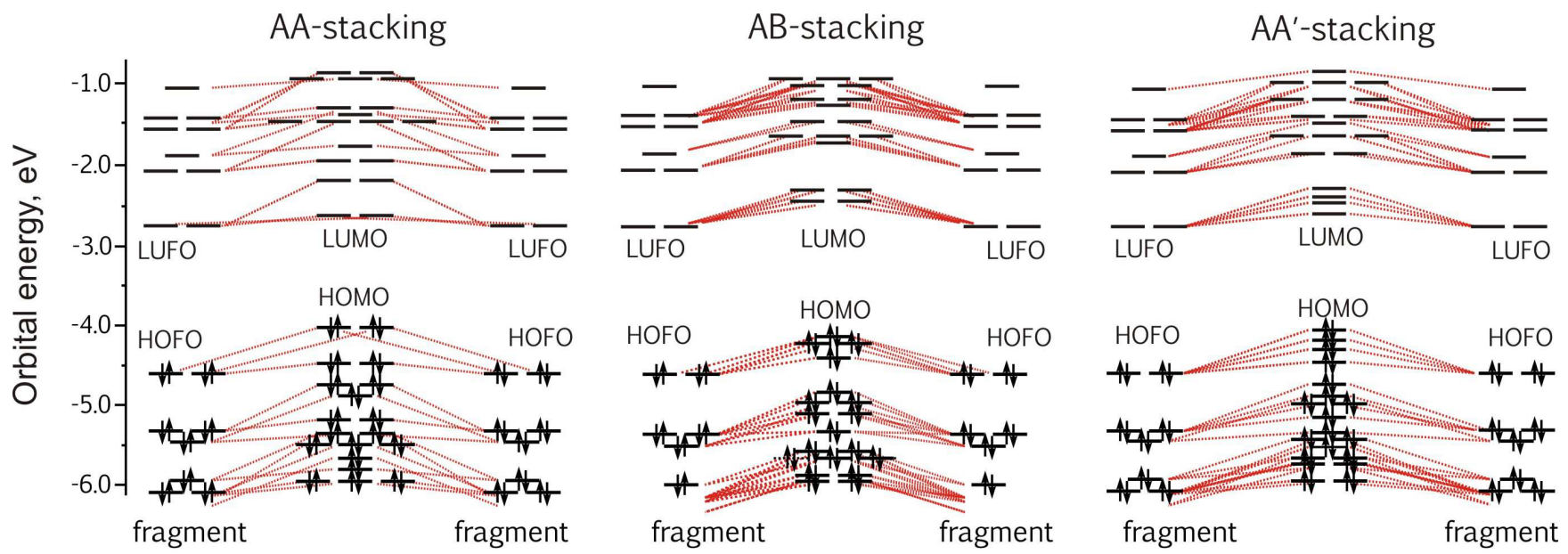

FIG. 2: The energy diagram demonstrating the formation of the molecular orbitals (HOMOs and LUMOs) as the fragment's orbitals overlap (HOFO and LUFO) for different arrangements of graphene lattices in the case when the fragments are separated by the equilibrium distance $d_{e q}$.

case must cause a significant suppression of the repulsive forces. To understand this, we considered the deviation of the bonding energy and its components with the change in the interlayer distance for the AA stacking [Fig. 1 (b)]. The negative sign is for the attractive interactions. The electrostatic interactions $\Delta V_{e l}$ and the orbital interactions $\Delta E_{o i}$ are both attractive in nature and their magnitudes are reduced with increasing distance between the fragments $\left(\Delta V_{e l}\right.$ can have a positive magnitude only for short interlayer distance when the nuclear repulsion dominates over other attractive terms). The contribution of the repulsive forces collected within $\Delta E_{p}$ into the total bonding energy displays its domination over the attractive terms only for a short interlayer distance, so the $\Delta E^{0}$ remains positive (repulsive) up to a distance of $3.2 \AA$. The bonding energy $\Delta E^{0}$ reaches its minimum at $d_{e q} \sim 3.67 \AA\left(\Delta E^{0} \sim-1.47 \mathrm{eV}\right)$ which is still energetically far from the ground state of $\mathrm{AB}$ stacking, where $\Delta E^{0}=-1.93 \mathrm{eV}$.

Among all the interaction terms the Pauli repulsion and the bonding interactions are points of particular interest. According to the Pauli principle the valence electron from one fragment is not supposed to penetrate the closed valence shell of the other fragment because the repulsive forces expel the charges from the overlap region. The main component within $\Delta E_{p}$ which contribute to the repulsion effect comes from the kinetic energy while the potential energy part is attractive [21]. It is noticeable in Fig. 1 (b) that as the fragments being separated at a distance beyond the value of $4.0 \AA$ the Pauli repulsion becomes negligible. However, for the interlayer distance $3.4 \AA$ which is typical for the AB stacking, the Pauli repulsion is still strong as $\Delta E_{p}=4.33 \mathrm{eV}$ while for the $\mathrm{AB}$ stacking pattern it was $3.38 \mathrm{eV}$. Therefore, if we compare the $\mathrm{AA}$ and $\mathrm{AB}$ stacking for the same interlayer distance $3.4 \AA$, the Pauli repulsion is larger by almost $1.0 \mathrm{eV}$ for the AA stacking, in agreement with our expectations, as stated above.

The orbital interaction energy $\Delta E_{o i}$ belongs to the attractive forces and its value reflects the efficiency of the donor-acceptor charge transfer between fragments which is controlled by the interlayer orbital overlap together with the Pauli repulsion and orbital polarization. Suppression of the Pauli repulsion with growing interlayer distance tends to increase the orbital interaction energy $\Delta E_{o i}$. However, a simultaneous reduction of the spatial orbital overlap generally leads to diminishing of $\Delta E_{o i}$, i.e., to a reduction of the charge transfer between the fragments. As a result, $\Delta E_{o i}$ being strongly attractive (negative sign) at short distances almost vanishes as the distance reaches the value of $d \simeq 3.3 \AA$ while after $d \simeq 3.9$ $\AA$ it turns repulsive with a positive sign. It was noticed that the composition of the molecular orbitals near the HOMO-LUMO gap is not changed with distance as it is shown in Fig. 3. For example, if the HOMO was formed by the interaction of selective HOFO orbitals provided by each fragment it remains of the same composition on a whole range of the distance while just become shifted in energy. Therefore, we can conclude that the orbital polarization term brings no contribution in deviation of $\Delta E_{o i}$ with distance.

To separate the orbital interactions from other forces, we calculated the spatial overlap integral between the fragments $J_{i, j}^{H-H}$ and $J_{i, j}^{H-L}$. The degradation of $J_{i, j}^{H-H}$ upon increase of the interlayer distance along with the size of the HOMO-LUMO gap $\left(E_{g a p}\right)$ are presented in Table II. Because a rise in interlayer separation $d$ induces a suppression of the orbital overlap, in particular of the overlap matrix $S_{i, j}$, the charge transfer integral $J_{i, j}^{H-H}$ is also being reduced. The same gradual reduction is observed for the $J_{i, j}^{H-L}$ overlap (from $5 \times 10^{-5}$ to $6 \times 10^{-6}$ $\mathrm{eV})$.

As one increases the interlayer distance $d$ the HOMOLUMO gap grows in contrast to the diminishing $J_{i, j}$ and 
TABLE II: The spatial overlap integral $J_{i, j}^{H-H}$ and HOMOLUMO gap $\Delta E_{\text {gap }}$ calculated for the AA-stacking pattern as the flake separation $d$ gradually increases. All values are in $\mathrm{eV}$.

\begin{tabular}{l|c|c|c|c|c|c|c|c}
\hline$d$ & 2.94 & 3.14 & 3.34 & 3.40 & 3.54 & 3.74 & 3.94 & 4.14 \\
\hline$J_{i, j}^{H-H}$ & -1.21 & -0.91 & -0.68 & -0.63 & -0.51 & -0.38 & -0.28 & -0.21 \\
$\Delta E_{\text {gap }}$ & 0.45 & 0.84 & 1.13 & 1.20 & 1.34 & 1.50 & 1.61 & 1.69 \\
\hline
\end{tabular}

its enhancement is directly connected to the orbital interaction between the fragments. We presented in Fig. 3 the energy diagram for the energetics of the $\pi$ and $\pi^{*}$ orbitals near the HOMO-LUMO gap for the case of separated fragments and their orbital splitting/mixing after perturbation. To find the orbital energy change by the fragment interaction we used the expression derived within the Hückel approximation for the description of splitting of the $\pi$ orbitals belonging to different fragments after inclusion of the interactions

$$
\begin{aligned}
& E_{1} \approx e_{0}+H_{12}-\left(e_{0}+H_{12}\right) S_{12} \\
& E_{2} \approx e_{0}-H_{12}+\left(e_{0}-H_{12}\right) S_{12}
\end{aligned}
$$

where $e_{0}$ and $E_{1,2}$ are the molecular $\pi$-orbital energies before (for identical graphene flakes $e_{1}=e_{2}=e_{0}$ ) and after perturbation, respectively. $S_{12}$ is the orbital overlap between the fragments and $H_{12}$ is the intrinsic interaction integral that is a combination of the energy terms responsible for electron-electron interactions and particularly the contribution of its repulsive part to the orbital energies $E_{1(2)}$.

As fragments are brought together to a distance of 2.94 $\AA$ (see Fig. 3) which is much shorter than the equilibrium separation $\left(d_{e q}=3.67 \AA\right)$, the repulsive force dominates over the attractive interaction. Therefore, since the electrons are expelled by repulsion from the overlap region, within the Hückel approximation the overlap matrix $S_{12}$ is treated as zero or would possess a negative value so that for simplicity, we can ignore the contribution from $\left(e_{0} \pm H_{12}\right) S_{12}$ to the orbital energy $E_{1(2)}$. Besides the effect of repulsion on $\left(e_{0} \pm H_{12}\right) S_{12}$ term, the strong repulsion between the fragments causes the increment of the $H_{12}$ and therefore, closer the fragments are to each other the larger the splitting of their $\pi$ orbitals. The significant $\pi-\pi$ splitting leads to shift of the LUMO and HOMO orbitals close to each other thus causing a suppression of the HOMO-LUMO gap. However, for a large interlayer distance of $4.14 \AA$, the value of $H_{12}$ diminishes because of the suppression of the Pauli repulsion, while the overlap matrix would have positive values. The charge exchange between the fragments is allowed which decreases the splitting $\left|E_{1}-E_{2}\right|$ for the $\pi$ orbitals and in turn enlarges the HOMO-LUMO gap $\left(\Delta E_{\text {gap }}\right)$.

In this section we considered the basics of the orbital interactions between two graphene fragments being under the control of the separation distance. It was gathered that there are two main components affecting the efficiency of the orbital interaction energy by varying the distance: the Pauli repulsion and the spatial orbital overlap, while no contribution from the orbital polarization was observed. The spatial orbital overlap decreases with increasing distance and so is the Pauli repulsion. These changes have the opposite influence on the orbital interaction energy $\Delta E_{o i}$, but since the decrease of the spatial orbital overlap is faster than the Pauli repulsion, $\Delta E_{o i}$ is generally reduced with induced flake separation.

\section{FLAKE ROTATION}

For two graphene layers stacked together, the rotational misorientation creates the Moiré pattern which is a periodic pattern manifesting itself through the spots where the superposition of the lattices is preserved, i.e., the AA stacking 11 13. The rest of the surface (the inter-spot regions) which are of a larger amount of the surface, possesses the stacking order similar or close to that for the $\mathrm{AB}$ arrangement (see Ref. 27] for images of various rotation structures). As the rotation angle increases, the percentage of AA-spotted areas grows while the size of the spots and the inter-spot areas shrink. Already for the angle above $20^{\circ}$ the spots with ideal AA stacking vanish completely, leaving the mixed and weakly defined interlayer lattice order. Therefore, the experimentally observed lattice misorientation induced by rotation angle of $3-5^{\circ}$ (see Refs. [1 [5]) is characterized by the well defined the AA-spots and of a high coverage of the $\mathrm{AB}$ stacking.

Graphene flakes of finite size are considered in this paper as a model system to represent the spots characterized by the lattice superposition (the AA-spots) in misoriented bilayer graphene. Therefore, by rotating one flake with respect to the other (the rotation axis is placed at the flake center) we basically recreate the modification of the shape of the AA-spots affected by the rotation angle. The main disadvantage of describing misoriented bilayer graphene by finite size flakes is to underestimate the contribution of the $\mathrm{AB}$ stacking areas to the electronic properties of the bilayer system. In the model system of rotated flakes the contribution of the inter-sport regions with $\mathrm{AB}$ stacking would depend on the flake size and therefore for small flakes would be negligible.

In a system of the AA stacked graphene fragments, the interacting $\pi$ orbitals between the fragments are perfectly orthogonal. The layer rotation observed in the experiments [1 [5] breaks that orthogonality, thereby modifying the balance of the attractive and repulsive forces between the layers. We simulated the effect of misorientation in the model system of two graphene flakes presented in Fig. 1 (a) with the rotation axis placed at the flake center. In a system of two flakes stacked in the AA pattern and separated by a distance of $d=3.4 \AA$ which is the equilibrium distance for the AB-stacking, we rotate one flake relative to the other for further elaboration of the interaction parameters. Our simulation results for 

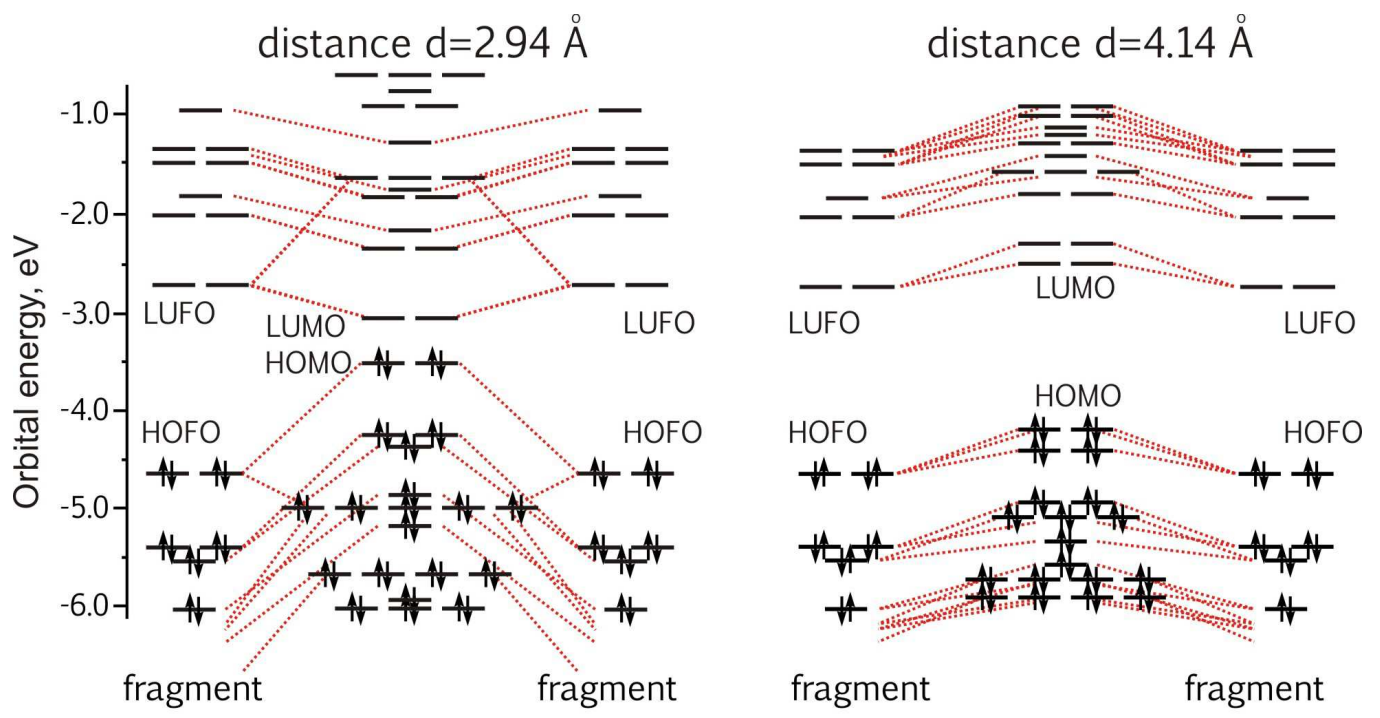

FIG. 3: Energetics of the $\pi$ and $\pi^{*}$ orbitals defining the HOMO-LUMO gap in the separated fragments and their splitting as fragments being stacked in the AA lattice arrangement.

TABLE III: The interlayer interactions in the system of two twisted flakes separated by a distance $d=3.4 \AA$. For the spatial overlap integral $J_{i, j}^{H-H}$, its modulus has been considered to avoid confusions of the sign change. $\Delta E_{g a p}$ is the HOMOLUMO gap in the system of two stacked flakes. For a better comparison we repeated the results for the graphene flakes stacked in the $\mathrm{AB}$ pattern at the equilibrium distance $d_{e q}=3.4$ $\AA$. All values are in $\mathrm{eV}$

\begin{tabular}{l|c|c|c|c|c|c|c}
\hline & $\Delta E_{\text {gap }}$ & $\left|J_{i, j}^{H-H}\right|$ & $\left|J_{i, j}^{H-L}\right|$ & $\Delta E_{o i}$ & $\Delta V_{e l}$ & $\Delta E_{p}$ & $\Delta E^{0}$ \\
\hline 0.0 & 1.203 & 0.627 & $1.6 \times 10^{-5}$ & -0.248 & -1.423 & 4.334 & -1.195 \\
1.5 & 1.209 & 0.604 & $2.5 \times 10^{-4}$ & -0.253 & -1.421 & 4.325 & -1.207 \\
3.0 & 1.226 & 0.544 & $6.5 \times 10^{-4}$ & -0.268 & -1.416 & 4.300 & -1.242 \\
6.0 & 1.287 & 0.563 & $6.5 \times 10^{-4}$ & -0.320 & -1.399 & 4.205 & -1.368 \\
9.0 & 1.365 & 0.305 & $8.9 \times 10^{-4}$ & -0.384 & -1.376 & 4.081 & -1.530 \\
12.0 & 1.436 & 0.116 & $2.3 \times 10^{-4}$ & -0.443 & -1.356 & 3.963 & -1.682 \\
15.0 & 1.478 & 0.131 & $6.5 \times 10^{-4}$ & -0.482 & -1.342 & 3.876 & -1.790 \\
18.0 & 1.492 & 0.061 & $6.9 \times 10^{-4}$ & -0.494 & -1.335 & 3.831 & -1.837 \\
20.0 & 1.489 & 0.176 & $3.9 \times 10^{-4}$ & -0.486 & -1.333 & 3.823 & -1.832 \\
22.0 & 1.485 & 0.318 & $1.1 \times 10^{-4}$ & -0.475 & -1.332 & 3.826 & -1.816 \\
24.0 & 1.481 & 0.184 & $5.8 \times 10^{-4}$ & -0.453 & -1.333 & 3.839 & -1.779 \\
26.0 & 1.478 & 0.344 & $3.7 \times 10^{-3}$ & -0.439 & -1.333 & 3.851 & -1.753 \\
28.0 & 1.477 & 0.363 & $2.0 \times 10^{-3}$ & -0.423 & -1.334 & 3.862 & -1.724 \\
30.0 & 1.476 & 0.322 & $2.9 \times 10^{-3}$ & -0.417 & -1.335 & 3.870 & -1.713 \\
AB & 1.568 & 0.229 & 0.169 & -0.482 & -1.184 & 3.388 & -1.931 \\
\hline
\end{tabular}

the bonding energy $\Delta E^{0}$ between layers and its components, overlap integrals such as $J_{i, j}^{H-H}$ and $J_{i, j}^{H-L}$, and the HOMO-LUMO gap $\left(\Delta E_{\text {gap }}\right)$ are displayed in Table [II] The results indicate that the size of the HOMO-LUMO gap $\left(\Delta E_{\text {gap }}\right)$ grows as the bonding interaction between layers is improved with rotation.

When two lattices of different flakes are superposed in the space, the electronic clouds of their $\pi$ orbitals are also superposed giving the maximum magnitude of the overlap integral $J_{i, j}^{H-H}$ which is being suppressed with the flake rotation because of misorientation of those $\pi$ clouds. Therefore, the spatial overlap integral $J_{i, j}^{H-H}$ reaches its minimum as the rotation angle reaches the value of $\theta \simeq$ $18^{\circ}$ which is being a result of significant disarrangement of the $\pi$ orbitals within the overlapping region from their superposed position. Another overlap integral accounting for inter-band interactions, $J_{i, j}^{H-L}$ (between HOMO and LUMO orbitals belonging to the different fragments), has shown the opposite behavior to that of $J_{i, j}^{H-H}$, i.e., with its minimum for the superposed case while growing with the flake rotation. However, even when $J_{i, j}^{H-L}$ reaches the maximum at $\theta=24^{\circ}-30^{\circ}$, its magnitude is still much lower than that found for the AB-stacking. For the angle in the range of $\theta=24^{\circ}-30^{\circ}$, both integrals $\left(J_{i, j}^{H-H}\right.$ and $\left.J_{i, j}^{H-L}\right)$ deviate insignificantly. With breaking of the orthogonality of the $\pi$ orbitals as the flake is rotated, the Pauli repulsion is also being suppressed by $\sim$ $0.5 \mathrm{eV}$ when its reaches its minimum at $\theta \simeq 18^{\circ}$. The reduced value of $3.831 \mathrm{eV}$ for $\theta \simeq 18^{\circ}$ becomes much closer to that for the AB stacking $\left(\Delta E_{p}=3.388 \mathrm{eV}\right)$.

Moreover, the orbital interaction energy $\Delta E_{o i}$ grows with flake rotation as the inter-band interactions reflected by the $J_{i, j}^{H-L}$ improve $\left(J_{i, j}^{H-L}\right.$ increases up to several orders of magnitude) along with the fast reduction of the interlayer repulsion. The coincidence of the minimum of $\Delta E_{o i}$ with the minimum value of the Pauli repulsion achieved for the $\theta \simeq 18^{\circ}$ is a clear evidence that the efficiency of the orbital interactions is being under direct control of the repulsive forces.

Therefore, because the layer rotation significantly suppresses the interlayer repulsion which in turn improves the orbital interaction, regardless of the deviation of the overlap integrals, the total bonding energy $\Delta E^{0}$ is lowered with rotation and its magnitude reaches its minimum also at $\theta \simeq 18^{\circ}$. In fact, the magnitude of 
the total bonding energy found for the angle $\theta \simeq 18^{\circ}$ $\left(\Delta E^{0}=-1.837 \mathrm{eV}\right)$ is comparable to that for the $\mathrm{AB}$ stacking $\left(\Delta E^{0}=-1.931 \mathrm{eV}\right.$ in Table I). Above the rotation angle $30^{\circ}$, some fluctuations for all the terms occur while closer to angle $60^{\circ}$ for which the conditions for the lattice superposition between two layers reappear, i.e., all the terms have the same values as for the angle $0^{\circ}$. Basically, the dependence in the range of the rotation angle from $30^{\circ}$ to $60^{\circ}$ is displayed in reverse order to that from $0^{\circ}$ to $30^{\circ}$.

Our main conclusion is that the repulsion appeared as result of the interaction of two systems of closed electron shells whose lattices are superposed, is the central force controlling the efficiency of the interlayer orbital interactions. As the flake rotation breaks the lattice superposition, the suppression of the repulsion induces an improvement of orbital interactions (such as the electronic coupling) thereby lowering the bonding energy $\Delta E^{0}$. Because the orbital interaction between the flakes depends on the flake rotation, the equilibrium distance $d_{e q}$ also fluctuates with rotation. The modification of the equilibrium distance $d_{e q}$ follows the dependence observed for the bonding energy $\Delta E^{0}$ which is controlled by the Pauli repulsion. The maximum equilibrium distance $d_{e q}=3.67$ $\AA$ is obtained for the AA stacking which is suppressed down to $d_{e q}=3.43 \AA$ as the flakes are at an angle $18^{\circ}$ that brings the system to the lowest energy state achievable with rotation. If the rotational angle grows further beyond $18^{\circ}$, the magnitude of $d_{e q}$ enhances again and for $\theta \simeq 30^{\circ}$ its value is $3.54 \AA$.

\section{DISCUSSION AND SUMMARY}

Two graphene layers stacked in the AA pattern which is characterized by the lattice superposition between the layers is the most unstable configuration in the bilayer geometry. The instability appears as a result of strong interlayer repulsion induced by the interaction of the filled orthogonal $\pi$ orbitals within the overlap region. Therefore, a disruption of the lattice superposition lowers the total bonding energy and therefore, leads to an enhancement of the system stability. The AB stacking is the most successful scheme to suppress the Pauli repulsion because it induces the maximum mis-orientation in the interlayer lattice order and therefore, the AB stacking appear to be the ground state of the system characterized by the strongest bonding interactions between the layers. However, an alternative way to induce the lattice mis-orientation from that superposed in the AA stacking and thereby, to transfer the system to the lower energy state is the layer rotation. It should be noted that any modification of the lattice order different from the ABstacking would be metastable (such as $\mathrm{AA}^{\prime}$ ) because $\mathrm{AB}$ stacking is the ground state of the system.

For adjacent graphene flakes of finite size, the rotation of one flake relative to the other induces a fast reduction of the repulsive part $\left(\Delta E_{p}\right)$ and an increase of the attractive forces (orbital interaction energy $\Delta E_{o i}$ ) such that both these tendencies lower the total bonding energy between the layers $\Delta E^{0}$. The lowest bonding energy $\Delta E^{0}$ is achieved for the rotation angle of $\theta \simeq 18^{\circ}$. This state is still metastable but with the lowest value of the bonding energy among all the rotation angles, and its value correlates with that for the AB-stacking being the ground state of bilayer graphene. However, as it was already noted above, the description of the adjacent graphene layers by the model system of finite flakes has crucial disadvantage caused by the underestimation of the inter-spot areas of the AB-stacking into the interlayer repulsion. As we switch to the twisted bilayer graphene of the infinite size, the contribution of large areas of $\mathrm{AB}$ stacking, which was largely neglected in the flake system, should be considered. For a small rotation angle the percentage of the AA spotted areas is large which decreases with angle enhancement. Thus, for an angle altered from $10^{\circ}$ to $12^{\circ}$, a decrease of $2.5 \%$ of AA staking is observed [27]. Obviously, since the larger interspot areas of AB-stacking is observed for small rotation angles $2^{\circ}-5^{\circ}$ (see Ref. [27] for the images of various rotation structures), we would expect that the rotation angle of much smaller magnitude than that for the finite systems might be required $\left(\theta<18^{\circ}\right)$ to bring the stacked graphene layers to the metastable state with the lowest energy.

However, additionally to rotation there is another way to make the system of the flakes stacked in the AA arrangement more stable which is to raise the interlayer distance when the bonding energy is lowered again due to suppression of the Pauli repulsion between the layers (see Fig. 1 (b)). In case of misoriented bilayer graphene exhibiting the Moiré pattern the distribution of the repulsive forces would be non-uniform as the lattice order is not the same in different areas, i.e. a maximum force pushing apart two lattices would originate at the AAspots of the lattice superposition. Moreover, another interesting distinction between the system of adjacent flakes and bilayer graphene of infinite size is alteration of its rigidity. Recalling that the free standing graphene is subjected to rippling of its surface [28], the lower rigidity of the graphene layers than that of flakes is anticipated. Therefore, we expect that a strong Pauli repulsion which is pronounced locally at the center of the AA-spots might not able to modify the interlayer separation throughout the whole system because of large areas of AB-stacking, but would rather induce a local lattice distortion forming the bump on the surface with its highest point at the center of the AA-spot [18]. To simulate this effect, the flake of larger size containing the bigger areas of AB stacking have been examined and already for that system the generation of the bump as high as $0.2 \AA$ was observed. However, the bump's height may be enhanced for an infinite system due to better efficiency of the attractive interactions in the inter-spot areas and lower rigidity of the layers. In fact, the appearance of bumps can explain the brightening of the AA-spots observed in the STM 
images of the twisted bilayer graphene $1-3]$.

The final point we wish to make is about the interlayer coupling which is found to be a function of the rotation angle and interlayer distance. As fragments being stacked, there are two types of $\pi$ interactions occurs, such as perturbation of the occupied/occupied orbitals $(\pi-\pi$ interaction) and interaction of the occupied/unoccupied orbitals $\left(\pi-\pi^{*}\right.$ interaction). According to the theoretical models developed to describe the behavior of the $\pi$ bands in bilayer graphene, the modification of the linear dispersion of the Dirac cones to a parabolic one has been simulated by inclusion only of the $\pi-\pi$ orbital interaction
7, 25]. However, we found that although the intra-bands interactions plays an important role, but particularly the inter-bands part must be introduced in the model to account for the decoupling effect arising in the AA stacked graphene layers. The orbital interaction energy $\Delta E_{o i}$ is therefore suppressed at least by five times as the lattice arrangement was changed from $\mathrm{AB}$ to $\mathrm{AA}$ due to vanishing of the $\pi-\pi^{*}$ interactions between fragments that is reflected by a drastic reduction of the spatial overlap integral $J_{i, j}^{H-L}$.

The work was supported by the Canada Research Chairs program.
[1] F. Varchon, P. Mallet, L. Magaud, and J.-Y. Veuillen, Phys. Rev. B 77, 165415 (2008).

[2] G. Li, A. Luican, J.M.B. Lopes dos Santos, A.H. Castro Neto, A. Reina, J. Kong, and E.Y. Andrei, Nature Phys. 6, 109 (2009).

[3] J. Hass, F. Varchon, J.E. Millán-Otoya, M.S. Prinkle, N. Sharma, W.A. de Heer, C. Berger, P.N. First, L. Magaud, and E.H. Conrad, Phys. Rev. Lett. 100, 125504 (2008).

[4] A. Reina, X. Jia, J. Ho, D. Nezich, H. Son, V. Bulovic, M.S. Dresselhaus, and J. Kong, Nano Lett. 9, 30 (2009).

[5] J.H. Warner,J.H. Warner, M.H. Rümmeli, T. Gemming, B. Büchner, and G.A.D. Briggs, Nano Lett. 9, 102 (2009).

[6] S. Shallcross, S. Sharma, E. Kandelaki, and O.A. Pankratov, Phys. Rev. B 81, 165105 (2010); S. Shallcross, S. Sharma, and O.A. Pankratov Phys. Rev. Lett. 101, 056803 (2008); J.M.B. Lopes dos Santos, N.M.R. Peres, and A.H. Castro Neto, Phys. Rev. Lett. 99, 256802 (2007).

[7] E. McCann, Phys. Rev. B 74, 161403 (2006).

[8] K.I. Bolotin, K.J. Sikes, Z. Jiang, M. Klima, G. Fudenberg, J. Hone, P. Kim, and H.L. Stormer, Solid State Commun. 146, 351 (2008).

[9] D.S.L. Abergel, V. Apalkov, J. Berashevich, K. Ziegler and T. Chakraborty, Adv. Phys. 59, 261 (2010).

[10] M.J. Allen, V. C. Tung, R.B. Kaner, Chem. Rev. 110, 132 (2010).

[11] S. Latil, V. Meunier, and L. Henrard, Phys. Rev. B 76, 201402 (2007).

[12] S. Shallcross, S. Sharma, E. Kandelaki, and O.A. Pankratov, Phys. Rev. Lett. 101, 056803 (2008).

[13] J.M.B. Lopes dos Santos, N.M.R. Peres, and A.H. Castro Neto, Phys. Rev. Lett. 99, 256802 (2007).

[14] M.S. Dresselhaus, and G. Dresselhaus, Adv. Phys. 30,
139 (1981).

[15] K. Yoshizawa, T. Yumura, T. Yamabe, S. Bandow, J. Am. Chem. Soc. 122, 11871 (2000); T. Yumura, K. Yoshizawa, Chem. Phys. 279, 111 (2002); K. Yoshizawa, T. Kato, T. Yamabe, Bull. Chem. Soc. Jpn, 71, 2087 (1998).

[16] H. Ruuska, and T.A. Pakkanen, J. Phys. Chem. 105, 9541 (2001).

[17] J.-C. Charlier, X. Gonze, and J.-P. Michenaud, Europhys. Lett, 28, 403 (1994).

[18] J. Berashevich and T. Chakraborty, Phys. Rev. B 84, 033403 (2011).

[19] S. Grimme, J. Comp. Chem. 25, 1463 (2004).

[20] ADF2008.01, SCM, Theoretical Chemistry, Vrije Universiteit, Amsterdam, The Netherlands.

[21] F.M. Bickelhaupt, and E.J. Baerends, Rev. Comp. Chem. 15, 1 (2000).

[22] K. Senthilkumar, F. C. Grozema, C. F. Guerra, F.M. Bickelhaupt, F. D. Lewis, Y. A. Berlin, M. A. Ratner, and L. D. A. Siebbeles, J. Am. Chem. Soc. 127, 14894 (2005).

[23] G.A. Shamov, P.H.M. Budzelaar, G. Schreckenbach, J. Chem. Theory and Comput. 6, 477 (2010).

[24] M. L. Senent, and S. Wilson, Int. J. of Quant. Chem. 82, 282 (2001).

[25] F. Zhang, B. Sahu, H. Min, and A. H. MacDonald, Phys. Rev. B 82, 035409 (2010).

[26] J.L. Bills and R. L. Snow, J. Chem. Educ. 59, 142 (1982).

[27] J.M. Campanera, G. Savini, I. Suarez-Martinez, and M.I. Heggie, Phys. Rev. B 75, 235449 (2007).

[28] A. Fasolino, J. H. Los and M. I. Katsnelson, Nature Materials 6, 858 (2007) 